University of Nebraska - Lincoln

DigitalCommons@University of Nebraska - Lincoln

1982

\title{
Stability Analysis of Numerical Boundary Conditions and Implicit Difference Approximations for Hyperbolic Equations
}

R. M. Beam

NASA Ames Research Center

R. F. Warming

NASA Ames Research Center

Helen C. Yee

NASA Ames Research Center, yee@nas.nasa.gov

Follow this and additional works at: http://digitalcommons.unl.edu/nasapub

Beam, R. M.; Warming, R. F.; and Yee, Helen C., "Stability Analysis of Numerical Boundary Conditions and Implicit Difference Approximations for Hyperbolic Equations" (1982). NASA Publications. 284.

http://digitalcommons.unl.edu/nasapub/284

This Article is brought to you for free and open access by the National Aeronautics and Space Administration at DigitalCommons@University of Nebraska - Lincoln. It has been accepted for inclusion in NASA Publications by an authorized administrator of DigitalCommons@University of Nebraska - Lincoln. 


\title{
Stability Analysis of Numerical Boundary Conditions and Implicit Difference Approximations for Hyperbolic Equations*
}

\author{
R. M. Beam, R. F. Warming, and H. C. Yee \\ NASA Ames Research Center, Moffett Field, California 94035
}

Received January 15, 1982

\begin{abstract}
Implicit noniterative finite-difference schemes have recently been developed by several authors for multidimensional systems of nonlinear hyperbolic partial differential equations. When applied to linear model equations with periodic boundary conditions those schemes are unconditionally stable (A-stable). As applied in practice the algorithms often face a severe time-step restriction. A major source of the difficulty is the treatment of the numerical boundary conditions. One conjecture has been that unconditional stability requires implicit numerical boundary conditions. An apparent counterexample was the space-time extrapolation considered by Gustafsson, Kreiss, and Sundström. In this paper we examine space (implicit) and space-time (explicit) extrapolation using normal mode analysis for a finite and infinite number of spatial mesh intervals. The results indicate that for unconditional stability with a finite number of spatial mesh intervals the numerical boundary conditions must be implicit.
\end{abstract}

\section{INTRODUCTION}

The boundary condition analysis described in this paper was motivated by the application of implicit finite difference algorithms to hyperbolic partial differential equations. As a simple example, consider the quasi-one-dimensional inviscid flow described by the gasdynamic equations in conservative form

$$
\frac{\partial U}{\partial t}+\frac{\partial F(U)}{\partial x}+H(U)=0 .
$$

A typical implicit algorithm (backward Euler in time) has the form

$$
\left[I+\Delta t\left(\frac{\partial}{\partial x} A^{n}+D^{n}\right)\right] \Delta U^{n}=-\Delta t\left(\frac{\partial F}{\partial x}+H\right)^{n},
$$

where $\Delta U^{n}=U^{n+1}-U^{n}, A=\partial F / \partial U, D=\partial H / \partial U$, and $\partial / \partial x$ is approximated by a three-point central difference operator. Consider the nozzle sketched in Fig. 1.1 with

* The one-step method results of this paper were presented at the SIAM 1981 National Meeting, Troy, New York, June 8-10, 1981. The general multistep results were presented at the Symposium on Numerical Boundary Procedures and Multigrid Methods, Moffett Field, California, October 19-22, 1981. 


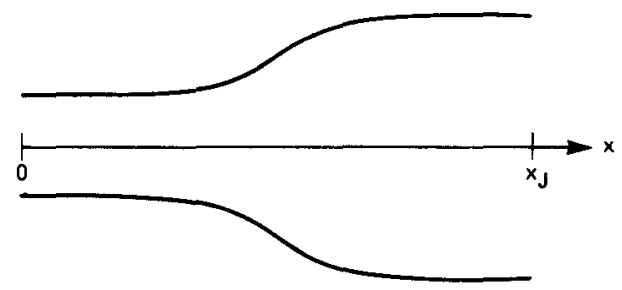

FIG. 1.1. Divergent nozzle $|1|$.

purely supersonic flow. For a well-posed problem, $U$ is specified on the inflow boundary $(x=0)$ and nothing is given on the supersonic outflow boundary. Algorithm (1.2) with central spatial differencing requires "numerical" boundary conditions on the right boundary at $x=x_{J}$. One way of providing the numerical boundary condition is by simply setting

$$
\Delta U_{J}^{n}=0
$$

on the right boundary and, after the interior solution is computed (by a block tridiagonal inversion), extrapolating the numerical solution to $J$

$$
U_{J}^{n+1}=U_{J-1}^{n+1} .
$$

For the backward Euler temporal differencing used in (1.2), procedure (1.3) is linearly equivalent to using zeroth-order space-time extrapolation

$$
U_{J}^{n+1}=U_{J-1}^{n}
$$

as the numerical boundary condition. There are two good reasons for using (1.3), or (1.4), as the numerical boundary scheme: it is extremely simple to implement and the interior scheme (1.2) with either (1.3) or (1.4) is stable for the initial-boundary-value problem according to a (linear) normal mode analysis of Gustafsson et al. [2].

To test the stability of scheme (1.2), (1.3) we computed the steady state solution to the nozzle flow (Fig. 1.1) using various CFL numbers. The number of time steps to converge is tabulated in Table I. We found the following peculiar results: Let $J$ be the number of spatial intervals. For an odd value of $J$ the scheme is unconditionally stable but if $J$ is even there is a finite stability limit. For even $J$, the stability limit depends on $J$. In addition, if we replace the zeroth-order extrapolation (1.4) by a linear space-time extrapolation

$$
U_{J}^{n+1}=2 U_{J-1}^{n}-U_{J-2}^{n-1},
$$

we obtain similar results but with a lower CFL limit (for the case of $J$ even).

The linear stability analysis of Gustafsson, Kreiss, and Sundström (GKS) [2] for the above problem is based on a theorem due to Kreiss [3] which relates the stability of the initial-boundary-value problem on a finite interval to the stability of the 
TABLE I

Zeroth-Order Space-Time Extrapolation

\begin{tabular}{|c|c|c|c|}
\hline \multicolumn{2}{|c|}{19 spatial intervals } & \multicolumn{2}{|c|}{20 spatial intervals } \\
\hline CFL & $\begin{array}{c}\text { Number of } \\
\text { steps to } \\
\text { converge }\end{array}$ & CFL & $\begin{array}{l}\text { Number of } \\
\text { steps to } \\
\text { converge }\end{array}$ \\
\hline 1 & 274 & 1 & 283 \\
\hline 10 & 33 & 5 & 50 \\
\hline 20 & 48 & 10 & 34 \\
\hline 30 & 46 & 15 & 52 \\
\hline 40 & 42 & 20 & 92 \\
\hline 50 & 56 & 22 & 141 \\
\hline 60 & 67 & 23 & 199 \\
\hline 70 & 77 & 24 & 353 \\
\hline 80 & 84 & 25 & 2072 \\
\hline 90 & 90 & 26 & does not converge \\
\hline $10^{2}$ & 93 & 27 & does not converge \\
\hline $10^{3}$ & 20 & & \\
\hline $10^{4}$ & 15 & & \\
\hline $10^{5}$ & 14 & & \\
\hline $10^{6}$ & 14 & & \\
\hline $10^{9}$ & 14 & & \\
\hline
\end{tabular}

difference approximation applied to the Cauchy problem and the related right and left quarter-plane problems. From the GKS-analysis it is clear that the results of a quarter plane problem cannot predict either the stability dependence on the number of mesh intervals (odd or even) or the dependence of the stability limit on the order of the space-time extrapolation.

In this paper we analyze the initial-boundary-value problem on the finite interval with a finite number of mesh points. This leads to a more restrictive definition of stability (than GKS-stability) which we call P-stability.

In Section 2 we give the difference approximations for the model scalar hyperbolic equation. We use a central spatial difference approximation and a linear multistep formula for the time integration. The extra numerical boundary condition is approximated by the $q$ th order space or space-time extrapolation. In Section 3 we review the definition of A-stability and define GKS-stability and P-stability. In addition, we delineate the normal-mode analysis for a quarter-plane problem.

Section 4 contains the stability analysis and the main results of this paper. We prove three theorems regarding the GKS-stability and P-stability of A-stable linear multistep methods and space or space-time boundary extrapolation. To simplify the proofs we make use of known properties of the stability regions of A-stable linear multistep methods. In the Appendix we present a more detailed analysis of the necessary and sufficient P-stability conditions for the class of one-step methods and 
space-time boundary extrapolation. The results show, for example, that the backward Euler method has a P-stability bound that depends on the number of spatial intervals: for an odd number of spatial intervals it is unconditionally P-stable; for an even number of spatial intervals it is conditionally P-stable with the bound being a function of the number of spatial intervals (Fig. 1.2). Our analysis explains the peculiar computational results of Table I.

We give a P-stability analysis for the class of all A-stable interior algorithms with all orders of space extrapolation as the numerical boundary condition, for example, zeroth-order space extapolation

$$
U_{J}^{n+1}=U_{J-1}^{n+1}
$$

and first-order space extrapolation

$$
U_{J}^{n+1}=2 U_{J-1}^{n+1}-U_{J-2}^{n+1} .
$$

We show that all of these schemes are unconditionally P-stable. As a numerical test, Table II presents results of the nozzle problem when (1.6) is used as the numerical boundary condition.

A more detailed discussion of the implementation of the boundary conditions for the equations of gas dynamics is presented in [1].

\section{Difference Approximations and Boundary Conditions}

In this paper we consider the numerical stability of finite difference approximations for the scalar hyperbolic initial-boundary-value problem

$$
u_{t}-c u_{x}=0, \quad 0 \leqslant x \leqslant l, \quad t \geqslant 0,
$$

where $c>0$. For a well-posed problem, initial data are given at $t=0$

$$
u(x, 0)=f(x), \quad 0 \leqslant x \leqslant l,
$$

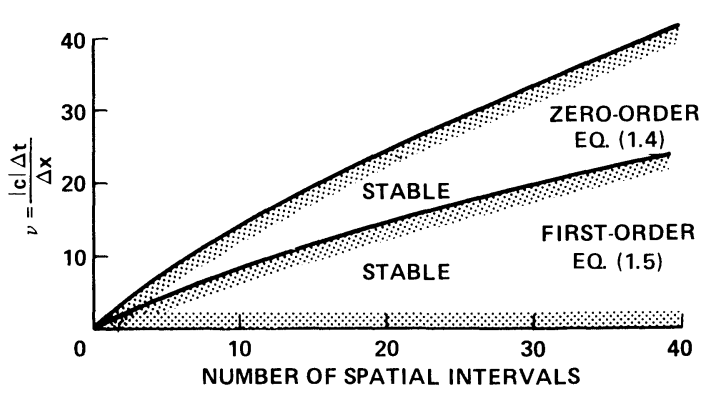

FIG. 1.2. P-stability bound for Euler implicit and space-time extrapolation (even number of spatial intervals). 


\section{TABLE II}

Zeroth-Order Space Extrapolation

\begin{tabular}{|c|c|}
\hline \multicolumn{2}{|c|}{20 spatial intervals } \\
\hline CFL & $\begin{array}{c}\text { Number of } \\
\text { steps to } \\
\text { converge }\end{array}$ \\
\hline 1 & 295 \\
\hline 10 & 28 \\
\hline 20 & 15 \\
\hline 30 & 11 \\
\hline 40 & 9 \\
\hline 50 & 8 \\
\hline 60 & 7 \\
\hline 70 & 7 \\
\hline 80 & 6 \\
\hline 90 & 6 \\
\hline $10^{2}$ & 6 \\
\hline $10^{3}$ & 4 \\
\hline $10^{4}$ & 4 \\
\hline $10^{5}$ & 4 \\
\hline $10^{6}$ & 4 \\
\hline $10^{9}$ & 4 \\
\hline
\end{tabular}

and boundary values are prescribed at $x=l$

$$
u(l, t)=g(t) .
$$

For the spatial mesh we divide the interval $0 \leqslant x \leqslant l$ into $J$ equally spaced intervals, $\Delta x=l / J$, i.e., $x=j \Delta x \quad(j=0,1,2, \ldots, J-1, J)$. We consider only the centered three-point spatial difference approximation to $\partial u / \partial x$ in $(2.1 \mathrm{a})$, i.e.,

$$
\frac{d u_{j}}{d t}-c \frac{u_{j+1}-u_{j-1}}{2 \Delta x}=0, \quad j=1,2, \ldots, J-1 .
$$

To integrate the first-order system of ordinary differential equations (2.2) we use a linear $k$-step formula defined by

$$
\rho(E) u^{n}=\Delta t \sigma(E)\left(d u^{n} / d t\right),
$$

where $\rho$ and $\sigma$ are the generating polynomials

$$
\rho(\zeta)=\sum_{i=0}^{k} \alpha_{i} \zeta^{i}
$$




$$
\sigma(\zeta)=\sum_{i=0}^{k} \beta_{i} \zeta^{i}
$$

and $E$ is the shift operator, i.e.,

$$
E u^{n}=u^{n+1} .
$$

In (2.3), $u^{n}$ is the numerical solution at $t=t^{n}=n \Delta t$ and $\Delta t$ is the time step. As an example, the most general consistent two-step formula (i.e., $k=2$ in (2.4)) can be written as

$$
(1+\xi) u^{n+2}-(1+2 \xi) u^{n+1}+\xi u^{n}=\Delta t\left[\theta{\frac{d u^{n+2}}{d t}}^{n+}+(1-\theta+\phi){\frac{d u^{n+1}}{d t}}^{n}-\phi \frac{d u^{n}}{d t}\right]
$$

where $(\theta, \xi, \phi)$ are arbitrary real numbers. The operators $\rho(E)$ and $\sigma(E)$ are

$$
\begin{aligned}
& \rho(E)=(1+\xi) E^{2}-(1+2 \xi) E+\xi, \\
& \sigma(E)=\theta E^{2}+(1-\theta+\phi) E-\phi .
\end{aligned}
$$

If we apply the linear $k$-step formula (2.3) to (2.2) we obtain

$$
\rho(E) u_{j}^{n}=\frac{c \Delta t}{2 \Delta x} \sigma(E)\left(u_{j+1}^{n}-u_{j-1}^{n}\right), \quad \begin{aligned}
j & =1,2, \ldots, J-1, \\
n & =k, k+1, k+2, \ldots .
\end{aligned}
$$

The values $u_{J}^{n}$ are obtained from the prescribed analytical boundary condition (2.1c), i.e.,

$$
u_{J}^{n}=g(n \Delta t), \quad n=1,2, \ldots
$$

To complete the computational algorithm we need, in addition to (2.8) and (2.9), a method for computing the values of $u_{j}^{n}$ at the boundary $j=0$, i.e., $u_{0}^{n}$; and a prescription for initial values $u_{j}^{n}, n=0,1, \ldots, k-1, j=1,2, \ldots, J-1$.

For the calculation of the boundary values $u_{0}^{n}$ we consider two extrapolation techniques: space extrapolation

$$
(F-1)^{q} u_{j}^{n}=0, \quad j=0, \quad q=1,2, \ldots ;
$$

and space-time extrapolation

$$
\left(F E^{-1}-1\right)^{q} u_{j}^{n}=0, \quad j=0, \quad q=1,2, \ldots,
$$

where $F$ is the spatial shift operator

$$
F u_{j}^{n}=u_{j+1}^{n}
$$


and $E$ is the temporal shift operator (2.5). The two lowest degree spatial extrapolations $(q=1,2)$ are simply zeroth- and first-order space extrapolation

$$
\begin{aligned}
& u_{0}^{n}=u_{1}^{n}, \\
& u_{0}^{n}=2 u_{1}^{n}-u_{2}^{n}
\end{aligned}
$$

or zeroth- and first-order space-time extrapolation

$$
\begin{aligned}
& u_{0}^{n}=u_{1}^{n-1}, \\
& u_{0}^{n}=2 u_{1}^{n-1}-u_{2}^{n-2} .
\end{aligned}
$$

The initial values $u_{j}^{0}$ are obtained from the analytical initial condition (2.1b), i.e.,

$$
u_{j}^{0}=f(j \Delta x), \quad j=0,1, \ldots, J .
$$

For higher-order $k$-step methods $(k>1)$ we assume that additional levels of initial data are given or they are calculated using an alternate numerical method.

In the following sections we investigate the numerical stability of the algorithm defined by (2.8), (2.9), and (2.10) or (2.11).

\section{Numerical Stability Definitions}

In this section we first review the definition of A-stable linear multistep methods. Next, we review the normal mode stability analysis of Kreiss [3] and Gustafsson et $a l$. [2] and define GKS-stability. Finally we add an additional constraint to the GKSstability and define what we believe is a stability definition applicable to many practical calculations.

\subsection{A-Stability}

If the linear $k$-step formula (2.3) is used to integrate the first-order ordinary differential equation

$$
d u / d t=f(u, t), \quad u(0)=u_{0},
$$

one obtains the linear multistep method (LMM)

$$
\rho(E) u^{n}=\Delta t \sigma(E) f^{n} .
$$

The linear stability of an LMM is analyzed by applying (3.1) to the linear test equation

$$
d u / d t=\lambda u
$$


where, in general, $\lambda$ is a complex constant. The stability region of an LMM consists of the set of all values of $\lambda \Delta t$ for which the characteristic equation

$$
\rho(\zeta)-\lambda \Delta t \sigma(\zeta)=0
$$

satisfies the root condition; that is, its roots $\zeta_{l}$ all satisfy $\left|\zeta_{l}\right| \leqslant 1$ and the roots of unit modulus are simple [4].

An LMM is said to be A-stable [5] if its stability region contains all of the left half of the complex $\lambda \Delta t$ plane including the imaginary axis. It can be shown [6] that a linear two-step method, i.e., (2.6), is A-stable if and only if

$$
\begin{aligned}
& \theta \geqslant \phi+\frac{1}{2}, \\
& \xi \geqslant-\frac{1}{2}, \\
& \xi \leqslant \theta+\phi-\frac{1}{2} .
\end{aligned}
$$

In this paper, for the stability analysis, we assume that temporal difference approximation (2.3) would produce an A-stable LMM. In part of the analysis a stronger stability definition is required.

An LMM is said to be strongly A-stable if:

(i) it is A-stable,

(ii) its stability boundary locus is tangent to the imaginary axis only at the origin, and

(iii) all roots of $\rho(\zeta)$ are inside the unit circle except for the root $\zeta=1$.

For example, the backward Euler $(\theta=1, \xi=0, \phi=0)$, second-order backward differentiation $\left(\theta=1, \xi=\frac{1}{2}, \phi=0\right)$, and Adams type $\left(\theta=\frac{3}{4}, \xi=0, \phi=-\frac{1}{4}\right)$ methods are strongly A-stable; however, the one-step trapezoidal rule $\left(\theta=\frac{1}{2}, \xi=0, \phi=0\right)$ does not satisfy condition (ii) and the two-step trapezoidal $\left(\theta=\frac{1}{2}, \xi=-\frac{1}{2}, \phi=-\frac{1}{2}\right)$ and the Lees type $\left(\theta=\frac{1}{3}, \xi=-\frac{1}{2}, \phi=-\frac{1}{3}\right)$ methods do not satisfy either condition (ii) or (iii).

\subsection{GKS-Stability}

Kreiss [3] has shown that the stability of a difference approximation for initialboundary-value problem (2.1) is related to the stability of the difference approximation applied to the initial-value, or Cauchy, problem

$$
\begin{aligned}
u_{t}-c u_{x} & =0, \quad-\infty<x<\infty, \quad t \geqslant 0, \\
u(x, 0) & =f(x)
\end{aligned}
$$


and to the related quarter-plane problems: the related right quarter-plane problem

$$
\begin{aligned}
u_{t}-c u_{x} & =0, \quad 0 \leqslant x<\infty, \quad t \geqslant 0, \\
u(x, 0) & =f(x)
\end{aligned}
$$

and the related left quarter-plane problem

$$
\begin{aligned}
u_{t}-c u_{x} & =0, \quad-\infty<x \leqslant l, \quad t \geqslant 0, \\
u(x, 0) & =f(x), \\
u(l, t) & =g(t) .
\end{aligned}
$$

Gustafsson et al. [2] developed a normal mode stability theory for general difference approximations to mixed initial-boundary-value problems, e.g., (3.5). For the purposes of this paper we make the following definition:

A difference scheme for an initial-boundary-value problem on a finite domain is said to be GKS-stable if it is stable (by the von Neumann ${ }^{1}$ method) for the Cauchy problem and stable (according to [2, Definition 3.3]) for the related left and right quarter-plane problems.

\subsubsection{Normal Mode Analysis}

In some of the proofs in Section 4 we rely on the previous work of Gustafsson and Oliger [7]; they provide a concise description of the application of the normal mode analysis. We repeat those parts required in the present analysis.

Results obtained by means of the normal-mode analysis are based upon the behavior of the so-called resolvent equations. These are formally derived (for the right quarter-plane problem) from (2.8) and (2.10), or (2.11), by substituting $u_{j}^{n}=z^{n} v_{j}$, where $z$ is a complex number. We obtain, respectively,

$$
\rho(z) v_{j}=(c \Delta t / 2 \Delta x) \sigma(z)\left(v_{j+1}-v_{j-1}\right)
$$

and

$$
(F-1)^{q} v_{j}=0
$$

or

$$
\left(F z^{-1}-1\right)^{q} v_{j}=0 .
$$

The general solution of (3.6) which is bounded as $j \rightarrow \infty$ for $|z|>1$ can be written in the form

$$
v_{j}=v_{0} \kappa^{j}
$$

\footnotetext{
${ }^{1}$ For the problems considered here, the von Neumann test is necessary and sufficient for the Cauchy problem.
} 
where $\kappa$ is the root of the characteristic equation

$$
\rho(z)=(c \Delta t / 2 \Delta x) \sigma(z)(\kappa-(1 / \kappa))
$$

such that $|\kappa|<1$ if $|z|>1$.

Equation (3.10) is formally obtained from (3.6) by substituting $v_{j}=\kappa^{j}$. Only one root of the quadratic in $\kappa,(3.10)$, has modulus less than one. This is an immediate consequence of the stability of (2.8) for the Cauchy problem (which is assured since the temporal integration scheme is assumed to be A-stable) and justifies (3.9). It is proved in [2, Lemma 10.3 and the following sentence] that the approximations are stable for the (right quarter-plane) initial-boundary-value problem if and only if (3.6) with boundary condition (3.7) or (3.8) has no nontrivial bounded solutions of form (3.9) for $|z| \geqslant 1$. (Note that one must include $|z|=1$.) This is established by substituting (3.9) into (3.7) or (3.8) and showing that $v_{0}=0$. When $|z|=1$, one or both of the roots of (3.10) may have modulus one. If this is the case, the $\kappa$ in (3.9) is defined by continuity to be that root which is the limit of the root $\kappa\left(z^{\prime}\right),\left|\kappa\left(z^{\prime}\right)\right|<1$ for $\left|z^{\prime}\right|>1$, as $\left|z^{\prime}\right| \rightarrow 1$. A nontrivial solution when $|z|>1$ and $|\kappa|<1$ is said to be an eigensolution and the corresponding $z$ an eigenvalue. A nontrivial bounded solution when $|z|=1$ and $|\kappa|=1$ is said to be a generalized eigensolution and the corresponding $z$ a generalized eigenvalue.

\subsection{P-Stability}

The stability analyses that provide GKS-stability bounds are very useful since they are relatively simple for scalar equations (with low order spatial difference approximations) and provide CFL limits that are directly applicable for many calculations which use explicit temporal difference approximations. They also provide a convenient method for eliminating undesirable numerical boundary schemes. They fail, however, to provide a sufficient stability condition for some practical calculations with implicit schemes. This is a result of the stability definition $[2$, Definition 3.3] which allows growing solutions if the mesh interval, $\Delta t$ or $\Delta x$, is not sufficiently sinall for a fixed value of $\Delta t / \Delta x$. Possible growing solutions for the classical wave equation are discussed in [2]. Therefore, it is desirable to have a more restrictive stability definition. We incorporate GKS-stability as a necessary condition and make the following definition:

A difference scheme for an initial-boundary-value problem is said to be Pstable if it is GKS-stable and all eigenvalues (corresponding to nontrivial eigenvectors) of the resolvent equations for a finite number of spatial mesh intervals have modulus less than or equal to unity.

The resolvent equations are obtained by substituting $u_{j}^{n}=z^{n} v^{j}$ into the difference approximation for the initial-boundary-value problem on the finite domain, e.g., (2.8), and the homogeneous boundary conditions, e.g., (2.9) with $g=0$, and (2.10) or 
(2.11). Note that these resolvent equations, which include both boundary conditions, differ from the resolvent equations in the GKS normal mode analysis, which includes only one boundary condition (in the analysis of each quarter-plane problem).

\section{Stability Analysis}

In this section we present our principal results. First we consider the space extrapolation boundary conditions and the general class of all A-stable temporal difference schemes. Next we consider the space-time extrapolation boundary condition for the more restricted class of strongly A-stable temporal schemes.

\subsection{Space Extrapolation Boundary Scheme}

We shall prove

THEOREM 4.1. Let $v=c \Delta t / \Delta x, c>0$. Algorithm (2.8), (2.9) and space extrapolation boundary scheme $(2.10)$ is $\mathrm{P}$-stable if the polynomials $\rho(E)$ and $\sigma(E)$ correspond to an A-stable LMM.

The GKS-stability for a limited class of two-step LMMs was investigated by Gustafsson and Oliger [7]. The extension to the general class of all A-stable LMMs requires only minor modifications which we consider here. As mentioned previously, the von Neumann stability of the Cauchy problem is assured since the temporal integration scheme is assumed to be A-stable. The GKS analysis of the left quarterplane problem is trivial. For the right quarter-plane problem we substitute (3.9) into boundary condition (3.7) and find that $v_{0}=0$ unless $\kappa=1$, which can only happen when $|z|=1$. Therefore, we need only check for a generalized eigenvalue and the generalization of the Gustafsson and Oliger analysis of the right quarter-plane problem requires only the following lemma:

Lemma 4.1. Assume the polynomials $\rho(z)$ and $\sigma(z)$ correspond to an A-stable LMM and $v>0$. The equation $\rho(z)=\frac{1}{2} v \sigma(z)[\kappa-(1 / \kappa)]$ has no solutions $|z|=1+\delta$, $\kappa=1-\varepsilon$, where $\delta>0, \varepsilon>0, \delta$ and $\varepsilon$ small.

Proof. Let $\kappa=1-\varepsilon, \quad \varepsilon>0$. Then $\quad \kappa-(1 / \kappa)=-2 \varepsilon+O\left(\varepsilon^{2}\right)$. Since $\operatorname{Re}\{v[\kappa-(1 / \kappa)]\}<0$ and the polynomials $\rho(z), \sigma(z)$ correspond to an A-stable LMM, it follows that $|z| \leqslant 1$, or $\delta \leqslant 0$.

We have proven GKS-stability. To complete the P-stability analysis and the proof of Theorem 4.1 we must prove that the eigenvalues of the resolvent equations have modulus less than or equal to unity. We obtain the eigenvalue equation by substituting $u_{j}^{n}=z^{n} \kappa^{j}$ into Eq. (2.8) and eliminating $\kappa$ by using homogeneous boundary conditions (2.9), with $g=0$, and (2.10). From (2.8) we obtain

$$
\rho(z)=\frac{1}{2} v \sigma(z)(\kappa-(1 / \kappa))
$$


which is a quadratic with two roots $\kappa,-\kappa^{-1}$. Let

$$
u_{j}^{n}=z^{n}\left[a \kappa^{j}+b(-(1 / \kappa))^{j}\right] \text {. }
$$

Boundary condition (2.9) with $g=0$ leads to

$$
0=a \kappa^{J}+b(-(1 / \kappa))^{J}
$$

or

$$
u_{j}^{n}=z^{n} a\left[\kappa^{j}-(-1)^{J} \kappa^{2 J}(-(1 / \kappa))^{j}\right]
$$

and the space extrapolation boundary condition (2.10) gives us an equation for $\kappa$, i.e.,

$$
(\kappa-1)^{q}-(-1)^{J+q} \kappa^{2 J-q}(\kappa+1)^{q}=0 .
$$

Note that $z$ does not appear explicitly in (4.4).

Remark. Polynomial (4.4) has $2 J$ roots and, at first glance, it appears that we have "too many" $\kappa$ 's for the size of the original linear system, i.e., $J-1$ unknowns at the interior points $j=1,2, \ldots, J-1$. It is easy to show that (4.4) has roots $\pm i$ which correspond to the trivial solution $u_{j}^{n}=0$ (Eq. (4.3)). There remain $2(J-1)$ distinct $\kappa$ roots. Recall that if $\kappa^{*}$ is a particular root so is $-1 / \kappa^{*}$, and both $\kappa^{*}$ and $-1 / \kappa^{*}$ give the same value of $(\kappa-1 / \kappa)$. Hence there are only $J-1$ distinct values of $(\kappa-1 / \kappa)$.

As a preliminary to the remaining proofs of this section we note the following. The characteristic equation (4.1) can be rewritten as

$$
\rho(z)-\lambda \Delta t \sigma(z)=0
$$

where

$$
\lambda \Delta t=\frac{1}{2} v(\kappa-(1 / \kappa)), \quad v>0
$$

(cf., (3.3)). An LMM is A-stable if and only if

$$
\operatorname{Re}(\rho(z) / \sigma(z))=\operatorname{Re}(\lambda \Delta t) \leqslant 0 \Rightarrow|z| \leqslant 1 ;
$$

therefore, since the LMM is assumed to be A-stable,

$$
\operatorname{Re}(\kappa-(1 / \kappa)) \leqslant 0 \Rightarrow|z| \leqslant 1,
$$

where $z$ denotes the roots of characteristic equation (4.5). Furthermore, from the identity

$$
\operatorname{Re}(\kappa-(1 / \kappa))=a\left(|\kappa|^{2}-1\right) /|\kappa|^{2}
$$


where $\kappa=a+i b,|\kappa|^{2}=a^{2}+b^{2}$, it follows that

$$
\left.\begin{array}{l}
|\kappa| \leqslant 1, \quad a \geqslant 0 \\
|\kappa|>1, \quad a<0
\end{array}\right\} \Leftrightarrow \operatorname{Re}(\kappa-(1 / \kappa)) \leqslant 0
$$

(see the shaded region of Fig. 4.1).

Before we examine the roots of polynomial ${ }^{2}(4.4)$ it is useful to have the following lemma:

Lemma 4.2. Assume the generating polynomials $\rho(z)$ and $\sigma(z)$ correspond to an A-stable LMM and $v>0$. If $|\kappa| \leqslant 1$ and $\operatorname{Re}(\kappa) \geqslant 0$, then all roots of polynomial (4.1) have modulus less than or equal to unity, i.e., $|z| \leqslant 1$.

Proof. The proof of the lemma follows from (4.10) and (4.8).

If $\kappa$ is a root of (4.4), then $-\kappa^{-1}$ is also a root; however, each produces the same value of $\kappa-(1 / \kappa)$. Therefore we need only consider value of $|\kappa| \leqslant 1$. To complete the proof of Theorem 4.1 we need only prove

Lemma 4.3. Polynomial (4.4) has no roots $\kappa$ with $|\kappa|<1, \operatorname{Re}(\kappa)<0$.

Proof. Rearrange the terms in (4.4)

$$
\kappa^{2 J-q}(\kappa+1)^{q} /(\kappa-1)^{q}=(-1)^{J+q} .
$$

The LHS of (4.11) has $2 J-q$ zeros at $\kappa=0, q$ zeros at $\kappa=-1$, and $q$ poles at $\kappa=1$ as shown in Fig. 4.2. The modulus of the LHS of (4.11) must equal unity if $\kappa$ is a root of (4.11), i.e., using the vectors defined in Fig. 4.2

$$
\left|r_{1}\right|^{2 J-q}\left|r_{2}\right|^{q} /\left|r_{3}\right|^{q}=1 \text {. }
$$

If $|\kappa|<1, \operatorname{Re}(\kappa) \leqslant 0$, then $\left|r_{1}\right|<1,\left|r_{2}\right| \leqslant\left|r_{3}\right|$; therefore $\kappa$ cannot be a root of (4.4). Consequently, the roots of (4.4) must fall in the shaded region of the $\kappa$-plane of
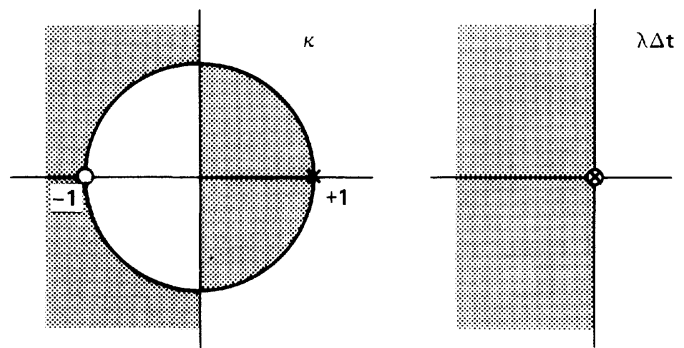

FIG. 4.1. Transformation $\lambda \Delta t=(v / 2)(\kappa-1 / \kappa)$.

${ }^{2}$ The eigenvalue equation would be formally obtained by solving (4.4) for $\kappa$ and eliminating $\kappa$ from (4.1) to obtain an equation in $z$. This procedure is neither practical nor necessary. 


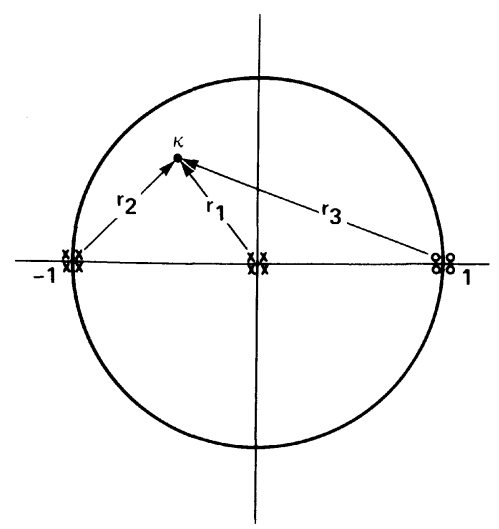

Fig. 4.2. $\kappa$ plane.

Fig. 4.1, and by (4.10) and (4.8) the eigenvalues of characteristic equation (4.1) have modulus $|z|<1$.

This completes the proof of Theorem 4.1.

\subsection{Space-Time Extrapolation Boundary Scheme}

For these boundary schemes it is convenient to first consider GKS-stability and prove

THeOREM 4.2. Let $v=c \Delta t / \Delta x, c>0$. Algorithm (2.8), (2.9) and space-time extrapolation boundary scheme (2.11) is GKS-stable if the polynomials $\rho(E)$ and $\sigma(E)$ correspond to a strongly A-stable LMM.

Again we present only those modifications of the Gustafsson and Oliger analysis [3] which are necessary to generalize their results to the class of all strongly A-stable LMMs. We substitute (3.9) into boundary condition (3.8) and find $v_{0}=0$ unless $z=\kappa$ which can only happen when $|z|=1$. Therefore we need only check for generalized eigenvalues and the modifications can be summarized by the following generalization of Lemma 4.1:

Lemma 4.4. Assume the generating polynomials $\rho(z)$ and $\sigma(z)$ correspond to a strongly A-stable LMM and $v>0$. The equation $\rho(z)=\frac{1}{2} v \sigma(z)[\kappa-(1 / \kappa)]$ has no solutions $|z|=1+\delta,|\kappa|=1-\varepsilon$, where $\delta>0, \varepsilon>0, \delta$ and $\varepsilon$ small, and $z \rightarrow \kappa$ when $\delta \rightarrow 0, \varepsilon \rightarrow 0$.

Proof. Since $\rho(z)$ and $\sigma(z)$ correspond to a strongly A-stable LMM, the stability region $(|z| \leqslant 1)$ includes the entire left half of the complex $\rho(z) / \sigma(z)$ plane plus a region to the right of the imaginary axis except at the origin (Fig. 4.3). All values of $|\kappa|=1$ lie on the imaginary axis of the $\kappa-(1 / \kappa)$ plane and values $|\kappa|=1-\varepsilon$ lie 


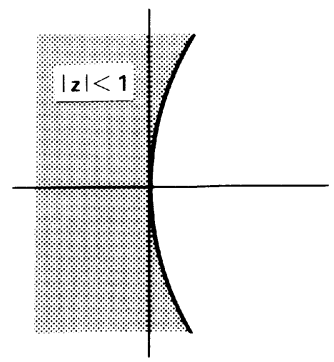

FIG. 4.3. $\rho(z) / \sigma(z)$ plane.

near the imaginary axis; but these values of $\kappa-(1 / \kappa)$ correspond to $|z| \leqslant 1$ (except at the origin) in the $\rho(z) / \sigma(z)$ plane, i.e., $|z|=1+\delta, \delta \leqslant 0$. The values $\kappa= \pm 1$ map into the origin of the $\kappa-(1 / \kappa)$ plane. Note that $z=1 \quad(\kappa=1)$ is not a generalized eigenvalue (by Lemma 4.1) but we must check $\kappa=-1$. Set $z=\kappa$, i.e., $\rho(z)=\frac{1}{2} v \sigma(z)[z-(1 / z)]$ and then $z=-1$ which implies $\rho(-1)=0$; however, -1 is not a root of $\rho(\zeta)$ since $\rho(\zeta)$ corresponds to a strongly A-stable LMM.

This completes the proof of Theorem 4.2.

Next we prove that the space-time extrapolation boundary scheme leads to a conditional P-stability bound.

THEOREM 4.3. Let $v=c \Delta t / \Delta x, c>0$. Assume $\rho(E)$ and $\sigma(E)$ correspond to a strongly A-stable LMM. For even values of J, algorithm (2.8), (2.9) and space-time extrapolation boundary scheme (2.11) has a necessary P-stability condition

$$
J \geqslant q \frac{\ln ((1+\kappa) /(1-1 / \kappa))}{\ln \left(\kappa^{2}\right)}
$$

where

$$
\kappa=[\rho(-1) / v \sigma(-1)]-\sqrt{ }[\rho(-1) / v \sigma(-1)]^{2}+1 .
$$

Remark. Inequality (4.13) implies a necessary conditional stability bound of the type sketched in Fig. 4.4.

We proceed as in the case of space extrapolation, i.e., by substitution of $u_{j}^{n}$ from (4.3) into boundary condition (2.11) which leads to

$$
(\kappa-z)^{q}-(-1)^{J+q} \kappa^{2 J}((1 / \kappa)+z)^{q}=0 .
$$

Note that, in contrast to (4.4) for space extrapolation, $z$ appears explicitly in (4.15). (Again we avoid the formal procedure of finding the eigenvalue equation which 


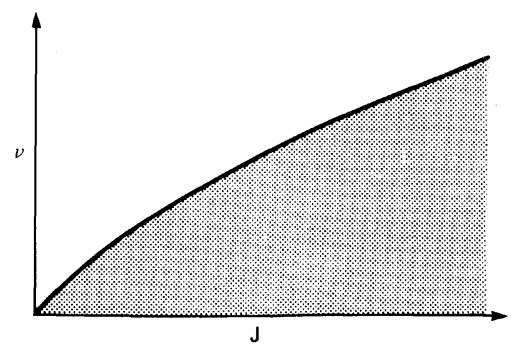

FIG. 4.4. Necessary stability region.

would require solving (4.15) for $\kappa(z)$ and eliminating $\kappa$ from (4.1).) Note that (4.15) can be rewritten

$$
J=\frac{\ln \left[(-1)^{J}((z-\kappa) /(1 / k+z))^{q}\right]}{\ln \left(\kappa^{2}\right)}
$$

and (4.1) can be rewritten $(v \neq 0, \sigma(z) \neq 0)$

$$
\kappa=[\rho(z) / v \sigma(z)]-\sqrt{ }[\rho(z) / v \sigma(z)]^{2}+1 .
$$

In order to prove that $(4.16)$ and (4.17) produce the necessary stability condition (4.13) and (4.14) when $z$ is set equal to -1 and $J$ is even, we seek solutions of (4.16) and (4.17) on the stability boundary, $z=-(1+\delta)$ as $\delta \rightarrow 0, \delta$ positive and real. Since $\rho(E)$ and $\sigma(E)$ correspond to an A-stable LMM

$$
\operatorname{Re}[\rho(z) / v \sigma(z)] \leqslant 0 \Rightarrow|z| \leqslant 1
$$

or conversely

$$
|z|>1 \Rightarrow \operatorname{Re}[\rho(z) / v \sigma(z)]>0 .
$$

Since $z=-(1+\delta)$ is real and $|z|>1$ we have

$$
\rho(-1-\delta) / v \sigma(-1-\delta)>0
$$

and from (4.17), as $v$ goes from $0^{+}$to $\infty, \kappa$ increases monotonically from $-1^{+}$to 0 , i.e., $-1<\kappa \leqslant 0$. For even values of $J$ and $z=-1$, (4.16) becomes

$$
J=\frac{\ln ((1+\kappa) /(1-1 / \kappa))^{q}}{\ln \left(\kappa^{2}\right)}
$$

and clearly for each value of $\kappa,-1<\kappa<0, J$ has a positive real value. From the derivative $d v / d J$ (obtained from Eqs. (4.13) and (4.14)) it is easily shown that $v(J)$ is a monotonically increasing function, e.g., Fig. 4.4. The direction of inequality (4.13) follows from GKS-stability of the scheme, i.e., for fixed $v$ no values $|z|>1$ as $J \rightarrow \infty$.

This completes the proof of Theorem 4.3 
Remark. Equations (4.13) and (4.14) provide a necessary P-stability condition. In the Appendix we consider necessary and sufficient P-stability conditions for the class of one-step methods.

\section{CONCluding Remarks}

In the analysis of the scalar hyperbolic initial-boundary-value problem, we have shown that if one combines the implicit space extrapolation boundary schemes with an unconditionally stable interior scheme, the combined scheme is unconditionally stable. If one combines the explicit space-time extrapolation boundary scheme with an unconditionally stable interior scheme, however, the combined scheme will be conditionally stable (if both odd and even numbers of mesh intervals are allowed) and the stability condition will depend on the number of mesh intervals. We have confirmed these results by numerical example for special cases of the quasi-onedimensional equations of gas dynamics.

\section{APPENDIX}

\section{P-Stability Conditions for One-Step Methods and Space-Time Extrapolation Boundary Scheme}

In Section 4.2 we found a necessary P-stability condition for the general A-stable LMM and space-time extrapolation boundary scheme (2.11). In this appendix we investigate the necessary and sufficient $\mathbf{P}$-stability conditions for the class of one-step methods.

The class of one-step or $\theta$ methods is $(\xi=\phi=0$ in (2.7) with the temporal index shifted down by one)

$$
\begin{aligned}
& \rho(E)=E-1, \\
& \sigma(E)=\theta E+(1-\theta)
\end{aligned}
$$

and they are A-stable if and only if (Eq. (3.4))

$$
\theta \geqslant \frac{1}{2} \text {. }
$$

GKS-Stability Analysis

The GKS-stability analysis for the one-step methods is included in the general analysis of Section 4. It is useful, however, to reexamine the details of the right quarter-plane analysis before procedding with the P-stability analysis.

From the analysis of the right quarter plane problem we have the characteristic equation (see (4.4))

$$
(z-1)=\frac{1}{2} v(\theta z+1-\theta)(\kappa-(1 / \kappa))
$$


and from space-time extrapolation boundary condition (2.11) the condition

$$
z=\kappa
$$

Now GKS-stability requires that there be no nontrivial solutions to (A.3) and (A.4) with $|z|>1,|\kappa|<1$. Substitution of $\kappa$ from (A.4) into (A.3) leads to

$$
(z-1)=\frac{1}{2} v(\theta z+1-\theta)(z-(1 / z))
$$

or if $z \neq 1(z=+1$ can be shown not to correspond to a generalized eigenvalue, see Fig. 4.1)

$$
z /[(z+1)(z+(1-\theta) / \theta)]=\frac{1}{2} v \theta
$$

The locus of the roots of (A.5) for all $v>0, \theta \geqslant \frac{1}{2}$ are shown in Fig. A1. The arrows indicate the direction of the motion of the roots as the parameter $v \theta$ changes from 0 to $\infty$.

The only possibility of a generalized eigenvalue occurs at the intersection of the root locus and the unit circle, $|z|=1$. We have already eliminated $z=+1$. Clearly, the only other possibilities are for $\theta=\frac{1}{2}$ where the locus lies on the unit circle or $z=-1$ for $\theta<\frac{1}{2}$.

Consider first $\theta=\frac{1}{2}$. Equation (A.3) becomes

$$
(z-1) /(z+1)=\frac{1}{4} v(\kappa-(1 / \kappa)) .
$$

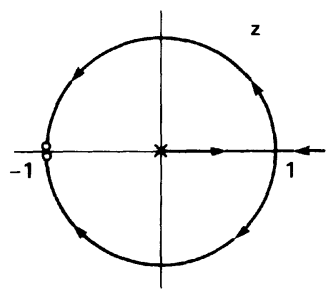

(a) $\theta=\frac{1}{2}$

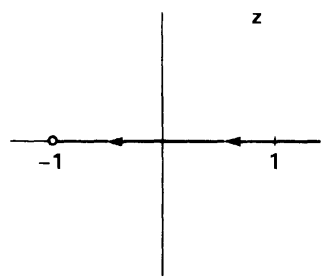

(c) $\theta=1$

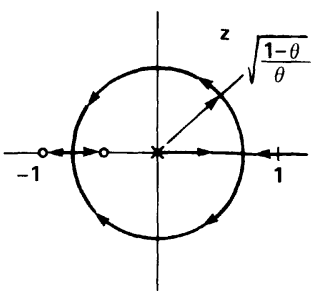

(b) $\frac{1}{2}<\theta<1$

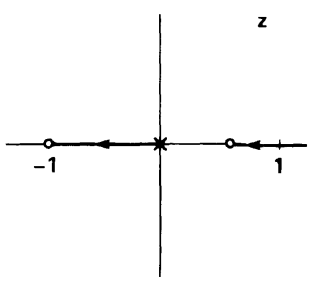

(d) $\theta>1$

Fig. A1. Locus of roots for Eq. (A.5). 
Next, we consider values of $\kappa$ near $z=\kappa=i(v=2)$. Let

$$
\kappa=(1-\varepsilon) e^{i(\pi / 2+\psi)}, \quad \varepsilon>0, \quad \psi>0,
$$

where $\varepsilon$ and $\psi$ are to be considered small. The RHS of (A.6) with $\kappa$ from (A.7) becomes

$$
\frac{1}{2}\left(\kappa-\frac{1}{\kappa}\right)=\frac{1}{2}\left[\frac{(1-\varepsilon)^{2}-1}{1-\varepsilon} \cos \left(\frac{\pi}{2}+\psi\right)+i \frac{(1-\varepsilon)^{2}+1}{1-\varepsilon} \sin \left(\frac{\pi}{2}+\psi\right)\right] .
$$

For small $\psi$

$$
\operatorname{Re}\left[\frac{1}{2}(\kappa-(1 / \kappa))\right] \approx\left[\left(-2 \varepsilon+\varepsilon^{2}\right) / 2(1-\varepsilon)\right](-\psi) .
$$

Thus for $\varepsilon$ small $(\varepsilon>0, \psi>0)$

$$
\operatorname{Re}\left[\frac{1}{2}(\kappa-(1 / \kappa))\right] \approx \varepsilon \psi>0 .
$$

Since $\theta=\frac{1}{2}$ corresponds to an A-stable LMM with none of the right half plane in its stability region, we know that a positive real part of the RHS of (A.6) implies $|z|>1$. Therefore, $z=\kappa=i(v=2)$ represents a generalized eigenvalue. It is easy to show that there is a generalized eigenvalue for all $v>2$ and no generalized eigenvalue for $v<2$. To summarize, if $\theta=\frac{1}{2}$ the scheme is GKS-stable for $v<2$. (The result is obvious from a comparison of Fig. Ala and Fig. 4.1.) Note that $\theta=\frac{1}{2}$ does not correspond to a strongly A-stable LMM and this result does not violate Theorem 4.2.

Next, we consider $\theta>\frac{1}{2}, v \rightarrow \infty$ (i.e., $z=-1$ ). Let

$$
z=-1-\delta, \quad \delta>0
$$

and substitute into (A.3)

$$
\kappa^{2}-(2(2+\delta) /((\delta \theta+2 \theta-1) v)) \kappa-1=0 .
$$

For large values of $v$

$$
\kappa \approx(2+\delta) /(\delta \theta+2 \theta-1) v \pm\left\{1+\frac{1}{2}[(2+\delta) /(\delta \theta+2 \theta-1) v]^{2}+\cdots\right\} .
$$

We choose the negative sign to ensure $\kappa \rightarrow-1$

$$
\kappa \approx-1+(2+\delta) /((\delta \theta+2 \theta-1) v)=-1+\varepsilon,
$$

where $\varepsilon>0$ and $\kappa \rightarrow-1^{+}$as $v \rightarrow \infty$. We conclude that $z=\kappa=-1$ is a generalized eigenvalue for $\lambda \rightarrow \infty$. There are, however, no generalized eigenvalues for $\lambda<\infty$ and the scheme is GKS-stable, in agreement with Theorem 4.2.

\section{P-Stability Analysis}

The necessary P-stability condition of Theorem 4.3, Eqs. (4.13) and (4.14), has (as the asymptotic limit $J \rightarrow \infty$ ) the generalized eigenvalue just considered in the GKS- 
stability analysis $(z=\kappa=-1)$. For the finite values of $J$ we no longer have the condition $z=\kappa$ and must consider the more complicated relation (4.15). To simplify the investigation we seek only the asymptote ( $J$ large) of the P-stability bound.

First we rewrite characteristic equation (A.3) and boundary condition polynomial (4.15), i.e.,

$$
(z-1) /(\theta z+1-\theta)=\frac{1}{2} v(\kappa-(1 / \kappa))
$$

and

$$
z=\left[(-1)^{J / q} \kappa^{-[(J / q)-1]}+\kappa^{(J / q)-1}\right] /\left[(-1)^{J / q} \kappa^{-(J / q)}-\kappa^{J / q}\right]
$$

and we shall assume $J \gg q$. Next we assume $J \gg 1$ and eliminate $\kappa$ from (A.12) and (A.13). For notational convenience let

$$
(z-1) /(\theta z+1-\theta)=\lambda * \Delta t .
$$

From (A.12) we have

$$
\kappa^{2}-(2 \lambda * \Delta t / v) \kappa-1=0
$$

or

$$
\kappa=(\lambda * \Delta t / v) \pm \sqrt{ }(\lambda * \Delta t / v)^{2}+1
$$

Recall

$$
v=c \Delta t / \Delta x=(c \Delta t / l) J
$$

therefore for large $J,($ A.15) becomes

$$
\kappa=1+(l \lambda * / c)(1 / J)
$$

Note that the choice of the sign before the radicand is arbitrary due to the special form of (A.12) and (A.13). We have chosen the positive sign. If we introduce $\kappa$ from (A.16) into (A.13) and assume $J \gg 1$, we obtain

$$
z=-\left[e^{l \lambda^{*} / q c}+(-1)^{J / q} e^{-\left(l \lambda^{*} / q c\right)}\right] /\left[e^{l \lambda^{*} / q c}-(-1)^{J / q} e^{-\left(l \lambda^{*} / q c\right)}\right]
$$

or

$$
e^{2 l \lambda^{*} / q c}=(-1)^{J / q}(z-1) /(z+1) .
$$

Thus, we have replaced $\kappa$ in (A.12) and (A.13) by $\lambda^{*}$ in (A.14) and (A.17). At the stability boundary $|z|=1$. We set $z=e^{i \psi}$ and substitute in characteristic equation (A.14)

$$
\lambda^{*}=\frac{2}{\Delta t}\left[\frac{(2 \theta-1) \tan (\psi / 2)+i}{(2 \theta-1)^{2} \tan (\psi / 2)+\operatorname{ctn}(\psi / 2)}\right]
$$


and boundary condition (A.17)

$$
\lambda^{*}=(c q / 2 l) \ln \left[i(-1)^{J / q} \tan (\psi / 2)\right] .
$$

For example, if we choose zeroth- or first-order space-time extrapolation, $q=1$ or 2 , (A.19) becomes

$$
\begin{aligned}
\lambda^{*} & =(q c / 2 l)[\ln (\tan (\psi / 2))+i((\pi / 2)+2 m \pi)], & & J / q=\text { even interger } \\
& =(q c / 2 l)[\ln (\tan (\psi / 2)+i((3 \pi / 2)+2 m \pi)], & & J / q=\text { odd integer } \\
& =(q c / 2 l)[\ln (\tan (\psi / 2))+i(\pi+2 m \pi)], & & J / q=\text { even integer }+\frac{1}{2} \\
& =(q c / 2 l)[\ln (\tan (\psi / 2))+i(2 m \pi)], & & J / q=\text { odd integer }+\frac{1}{2}
\end{aligned}
$$

where $m=0, \pm 1, \pm 2, \ldots$.

Symbolically we can write (A.18) and (A.19)

$$
\begin{aligned}
& \lambda_{\mathrm{CE}}^{*}=(1 / \Delta t) f(X), \\
& \lambda_{\mathrm{BC}}^{*}=(q c / l) g(X),
\end{aligned}
$$

where $X=\tan (\psi / 2)$ and $f$ and $g$ are complex functions of $X$. The subscripts CE and $\mathrm{BC}$ on $\lambda^{*}$ have been added for notational convenience; of course, $\lambda_{\mathrm{CE}}^{*}$ must equal $\lambda_{\mathrm{BC}}^{*}$.

We have found the following procedure to be convenient for solving (A.21): Choose $\theta$. Equate the arguments of $\lambda_{\mathrm{CE}}^{*}$ and $\lambda_{\mathrm{BC}}^{*}$, i.e.,

$$
\operatorname{Arg}\left(\lambda_{\mathrm{BC}}^{*}\right)=\operatorname{Arg}\left(\lambda_{\mathrm{CE}}^{*}\right)
$$

or

$$
\operatorname{Arg}(f(X))=\operatorname{Arg}(g(X)),
$$

where (A.22) is a nonlinear equation in $X$ which can easily be solved by Newton's method. Next equate the absolute values of $\lambda_{\mathrm{CE}}^{*}$ and $\lambda_{\mathrm{BC}}^{*}$, i.e.,

$$
\left|\lambda_{\mathrm{CE}}^{*}\right|=\left|\lambda_{\mathrm{BC}}^{*}\right|
$$

or

$$
(1 / \Delta t)|f(X)|=(q c / l)|g(X)|
$$

or, since $v=c \Delta t / \Delta x, \Delta x=l / J$,

$$
v=(J / q)|f(X) / g(X)|,
$$

which is an asymptote for the stability boundary, $|z|=1$. In general, there can be more than one asymptote since $g(X)$ is a function of $m$, e.g., Eq. (A.20). The Pstability condition is the most restrictive condition (minimum $v$ ) obtained from the set 
(A.23) and (4.13), (4.14). Domains of P-stability for typical methods with the spacetime extrapolation boundary scheme $(J$ even, $q=1)$ are shown in Fig. A2.

For some values of $\theta$ and $q$ there are no asymptotes (A.23). For example, let $q=1$ and $J$ be even. We have from (A.18)

$$
f(X)=[(2 \theta-1) X+i] /\left[\left(2 \theta^{2}-2 \theta\right) X+\frac{1}{2}(X+(1 / X))\right]
$$

and from (A.20a)

$$
g(X)=\frac{1}{2}[\ln (X)+i((\pi / 2)+2 m \pi)] ;
$$

therefore (A.22) becomes

$$
1 /((2 \theta-1) X)=(\pi / 2+2 k \pi) / \ln (X) .
$$

Equation (A.24) has no solution $\left(\theta>\frac{1}{2}\right)$ if

$$
\theta>\frac{1}{2}\left[1+\left(1 / \pi e\left(\frac{1}{2}+2 m\right)\right)\right] .
$$

Therefore, if

$$
\theta>\frac{1}{2}+(1 / \pi e) \approx 0.6171
$$

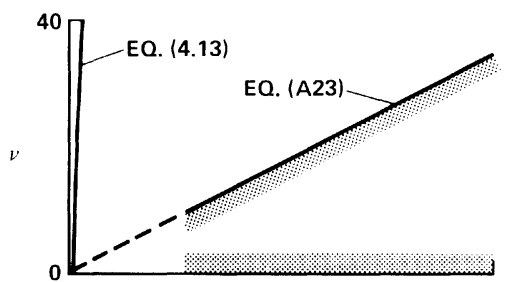

(a) $\theta=0.52$

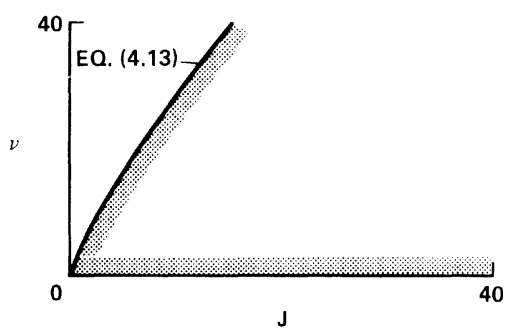

(c) $\theta=0.75$

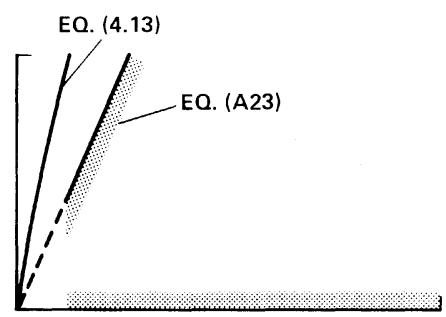

(b) $\theta=\mathbf{0 . 6 0}$

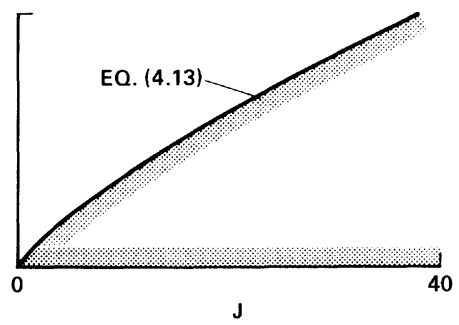

(d) $\theta=1.0$

FIG. A2. P-stability domain (shaded region) for typical $\theta$ methods, space-time extrapolation boundary condition, $J$ even, $q=1$. 
there are no asymptotes (A.23) and the P-stability bound is (4.13), (4.14). Similarly, if $q=1$ and $J$ is odd there are no asymptotes (A.23) if

$$
\theta>\frac{1}{2}+(1 / 3 \pi e) \approx 0.5390
$$

and the schemes are unconditionally P-stable. Note that the values (A.25) and (A.26) were derived for large $J$ and may not apply for $J$ near unity.

\section{REFERENCES}

1. H. Yee, R. Beam, and R. Warming, in "Proceedings, AIAA Computational Fluid Dynamics Conference, Palo Alto, California, June 22-23, 1981," Paper No. AIAA-81-1009-CP.

2. B. Gustafsson, H.-O. Kreiss, and A. Sundström, Math. Comp. 26 (1972), 649.

3. H.-O. KreIsS, in "Proceedings of Advanced Symposium, Madison, Wisconsin, 1966," pp. 141-166, Wiley, New York.

4. C. Gear, "Numerical Initial Value Problems in Ordinary Differential Equations," Prentice-Hall, Englewood Cliffs, N.J., 1971.

5. G. Dahlquist, BIT 3 (1963), 27.

6. R. Beam and R. Warming, in "Proceedings, AIAA 4th Computational Fluid Dynamics Conference, Williamsburg, Virginia, July 1979," Paper No. 79-1466.

7. B. Gustafsson and J. Oliger, "Stable Approximations for a Class of Time Discretizations of $u_{t}=A \dot{D}_{0} u$," Report No. 87, Uppsala University, Department of Computer Sciences, 1980. 